\title{
The cancer agenda: revisited
}

\section{Recnac 2 project}

Cancer is one of the main killers in our society, in addition of being responsible of many deaths; it is also responsible for much physical and emotional suffering even in those who survive and their loved ones. In addition, to increasing morbidity, mortality and suffering, there is an enormous economic impact. In one side, it can drain the economy of families and force them to assume very significant debts, and depending on the public policy, it can consume significant part of the health budget of cities and even countries. Interestingly enough, while there are very significant costs to individuals, families and government, it can bring enormous profits to certain companies in the health industry.

Articles on newspaper, WebPages, radio programs, TV shows and so on often bring prominent oncology specialists and scientist in prestigious institutions to discuss the cancer agenda and despite being sophisticated in their discussion, most of the time they keep nourishing new elements within the same paradigm that had brought us to the current situation of increasing incidence and prevalence and similar mortality as 55-60 years ago.

\section{Myths of the old paradigm}

a. Cancer is not one disease, but a group of diseases.

b. Cancer is a genetic disease.

c. Early detection is Prevention.

d. Radiation and Chemotherapy is a rational first line treatment.

e. Environmental risks are basically/mainly tobacco, obesity and aging.

\section{New paradigm}

I. Cancer is based on an energy problem within the cell, and its manifestation depends on the particular tissue that it arises and the causative/ contributing factors. Cancer is one disease with multiple expressions.

II. Cancer is not a genetic disease, it's a metabolic disease were gene expression is affected secondary to an energy (metabolic) problem that induces the cell to a survival mode (Survival devolution).

III. Early detection is not prevention because if detected cancer is already present. Prevention is the detection of the biochemical conditions that favors abnormal growth (malignancy) before the establishment of the malignant growth. This includes markers of cellular proliferation, detection of toxins in body tissues and fluids and determination of nutrient plasma levels. Prevention is intervention at a much earlier stage, the earliest the better. This allows correcting biochemical abnormalities that are known or suspected to be precursors of cancer. Changing the physiological environment from one that promotes cancer to one that promotes healthy tissues is real prevention.

IV. Another problem is that many diagnostic tools expose the patients to a substantial amount of radiation that is known to cause cell,
Volume I Issue 4 - 2014

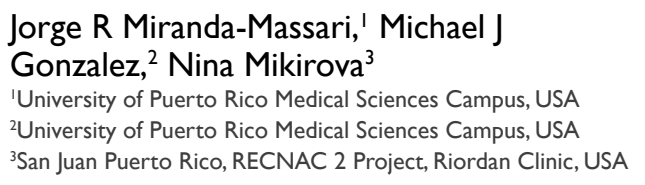

Correspondence: Jorge R Miranda- Massari, University of Puerto Rico Medical Sciences Campus, School of Pharmacy, San Juan Puerto Rico, USA, Tel 787-758-2525, Fax 787-759-67I9, Email Jorge.miranda2@upr.edu

Received: October 23, 2014 | Published: October 25, 2014

membrane and DNA damage, that if not repaired could lead to neoplasia.

V. Chemotherapy and radiation kill not only cancer cells but healthy cells including the immune system cells necessary to control cancer. In addition chemotherapy and radiation promote cell, membrane and DNA damage which are proposed to be causes of cancer.

The first rational therapy would be to correct the cause and chemo and radiation does not do that.

A. Important Risk factors that induce energy problems:

B. Inappropriate diet too rich in simple and refined carbohydrates.

C. Inappropriate diet too low in co-factors necessary for energy production and sustained metabolism.

D. Low oxygenation.

E. Low $\mathrm{pH}$.

F. Presence of contaminants (pesticides, tobacco, radiation, medications, hydrocarbons, heavy metals, nitrates, alflatoxins, halogens and others).

\section{Optimal cancer public policies}

1. Government taxes tobacco and alcohol heavily because this products cause significant health problems with significant society cost. Tax monies help deal with these costs. The similar policies should apply to other products that pose significant health risks with significant costs such as soft drinks, refined products, pesticides, hydrocarbons, heavy metals and others.

2. Accordingly, incentives should be given to the products that contribute to nourishment, health and improvement in the quality of life. 


\section{The sugar tax issue}

Sugar drinks are one of the largest contributors of excess empty calories in our nation's diet. A growing body of evidence suggests that reducing consumption of these drinks could be the single most effective intervention in reducing the prevalence of obesity in the United States. (Probably Cancer, Heart Disease and Diabetes too!). In the United States, sugar drinks account for approximately one-half of the recent increased calorie consumption, represent the largest source of added sugars and are one of the single most significant sources of energy contributing to excess calorie consumption.

States and localities have begun to consider taxing sugarsweetened beverages; including sodas, sports drinks, sweetened tea, fruit drinks and punches, and other sweetened beverages, in order to generate revenue, reduce consumption of unhealthy beverages and Promote public health. Research on several natural substances and/or non-toxic substances such as intravenous vitamin $\mathrm{C}$ has demonstrated activity against cancer cells and clinical studies have demonstrated that it can sustain or improve the results from other treatments while improving quality of life.
We have to give more importance to the quality and purity of the air we breathe to the water we drink and to the food we eat. Treatments that can correct the fundamental causes will be more effective (survival) and less toxic (quality of life). New developments are allowing interventions that also improve quality of life. Only by changing our paradigms and putting it into practice we can change outcomes. We need to improve in order to decrease morbidity and mortality which has not been significantly impacted in the last 50 years!

\section{Acknowledgments}

None.

\section{Conflicts of interest}

Authors declare there are no conflicts of interest. 